\title{
Search Tools that Improve Portability Anti- Tuberculosis Drugs in Experimental Conditions
}

\author{
Usov KI* \\ Research Institute Biophysics, Federal state budgetary educational institution of higher education, Russia \\ *Corresponding author: Usov KI, Research Institute Biophysics, Federal state budgetary educational institution of \\ higher education, 665835, Partizanskaya street, Angarsk, Russia
}

\section{ARTICLE INFO}

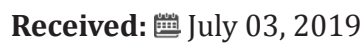

Published: 幽 July 11, 2019

Citation: Usov KI. Search Tools that Improve Portability Anti-Tuberculosis Drugs in Experimental Conditions. Biomed J Sci \& Tech Res 19(4)-2019. BJSTR. MS.ID.003320.

\begin{abstract}
The scientific report contains information on the search for drugs that improve the tolerability of anti-TB drugs. The data of experimental substantiation of the combined use of anti-TB drugs and ademetionine as a means of maintenance therapy are presented. The influence of chronobiological rhythms on the toxicity of isoniazid and rifampicin is shown. When studying the effect of sucrose on the toxicity of isoniazid, it was found that its appointment after a meal with the addition of sugar to the diet improves the tolerability of isoniazid.
\end{abstract}

Keywords: Tolerability of Anti-TB Drugs; toxicity; Side Effects; Chemotherapy of Tuberculosis; Hepatoprotector

\section{Introduction}

One of the main reasons for the insufficient effectiveness of tuberculosis chemotherapy, along with the increasing drug resistance of the pathogen and low patient compliance, is the high incidence of adverse side effects (NPE) of anti-tuberculosis drugs (PTP). Cancellation of PTP due to the manifestation of severe NPE is made in 20-91\% of patients with tuberculosis. Drug load significantly increases in patients with comorbidities that form a cycle are inevitable problems of pharmacotherapy: a "polymorbidity" $\rightarrow$ "polypragmasia" $\rightarrow$ "NPE". One of the ways to solve the problems associated with the manifestation of severe NPE for the use of PTP in humans is to find means to improve their portability. The purpose of the study: to find means to improve the tolerability of PTP.

\section{Materials and Methods}

White nonlinear rats were used as an experimental biological model. The animals were kept in a specialized laboratory animal clinic (vivarium) - veterinary certificate 238 № 0019883. The experiments were conducted in accordance with the ethical requirements for working with laboratory animals [1] and allowed by the local ethical Committee. The drugs were administered intragastrically as a suspension in distilled water, using a metal atraumatic probe. The development of approaches aimed at reducing NPE PTP, conducted in 3 directions: a) Rational determination of the time of reception of PTP, which examined the influence of circadian rhythms on the toxicity of the PCP, namely: group 1 rats were administered PCP at 10 am, group 2 in 22 hours in the evening. Isoniazid was administered in doses of $0.6 \div 2.4 \mathrm{~g} / \mathrm{kg}$ with a step of increasing the dose by $0.1 \mathrm{~g}$, rifampicin - in doses of $6.0 \div 24 \mathrm{~g} / \mathrm{kg}$ with a step of increasing the dose by $1 \mathrm{~g}$;

b) Determination of the role of food consumption in the tolerability of PTP, which was studied with the introduction of isoniazide on an empty stomach (deprivation of animal feed 14 hours before administration of drugs and 1 hour before administration of PTP); studied the effect of sucrose on the toxicity of isoniazide administered in the average dose (DL50 = $1233 \pm 43 \mathrm{mg} / \mathrm{kg}$ [2]);

c) Study of the possibility of using hepatoprotector to reduce hepatotoxicity of PTP, for which in the experimental conditions 2 schemes of drug administration were used: one of the schemes was a two - stage joint administration of the studied drugs-the first stage was the introduction of PTP in toxic doses: isoniazid at a dose of $240 \mathrm{mg} / \mathrm{kg}$ (1/5 of DL50,) pyrazinamide at a dose of $380 \mathrm{mg} / \mathrm{kg}$ (1/10 of DL50), rifampicin at a dose of $950 \mathrm{mg} /$ $\mathrm{kg}$ (1/20 of DL50), starting from 10 am and until the clinical detection of drug complications in the form of manifestations 
of severe adverse hepatotoxic reactions of the body, followed by cancellation of PTP; the second stage - the appointment of the drug ademetionine (AM) on the next day after the abolition of PTP.

AM was used at a dose of $120 \mathrm{mg} / \mathrm{kg}$ [3], hepatoprotector was administered 2 hours before the introduction of PTP. The second scheme was a one-step combined application of AM with PTP. The use of AM in the second scheme started with the first day of the introduction of PTP. In the serum of animal blood, obtained by decapitation, was determined by the content of enzymes: an alanine aminotransferase (ALT), aspartataminotransferase (AST), gammaglutamyltranspeptidase, urokinase, alkaline phosphatase, phospholipase A. Of clinically relevant biochemical parameters were determined: total protein, total cholesterol, total and direct bilirubin, glucose. The activity of ALT and AST were determined by the micro method in homogenized liver tissue with subsequent spektrofotometricheskoe determination of optical density and calculation of concentration in mol/L. For processing the obtained results were used methods of mathematical statistics, differences were considered significant when $p \leq 0.05$ [4-6].

\section{Results}

Introduction in rats, a median lethal dose of isoniazid to 10 a.m., and 22 hours is allowed to determine the decrease of toxicity of the drug when administered in the evening hours - increase of DL50 was $12 \%$, and the average life expectancy of rats increased in 2 times. A joint single administration of isoniazide in a mediumdose dose with rifampicin at different times of the day also led to a decrease in the toxicity of isoniazide and an extension of the average duration of animals receiving drugs in 22 hours. There was a decrease in the effects of neurotoxicity of isoniazid. The results indicate the need to take into account the body's biorhythms in the appointment of PTP [4]. Given that PTP are carried by rats, leading a night life, it is better when administered in the evening, ie, against the background of their maximum activity, patients should be recommended to use PTP in the morning against the background of maximum human activity.

The introduction of isoniazid on an empty stomach (deprivation of animal feed 14 hours before the introduction of drugs) led to greater toxicity, compared with the introduction of the drug to experimental rats deprived of feed for 1 hour before its introduction. The introduction of drugs on an empty stomach led to a reduction in the average life expectancy of experimental rats by $79 \%$. The use of isoniazide with sugar syrup led to $100 \%$ survival of experimental rats regardless of the duration of fasting animals. The results of the study showed that taking isoniazid after eating improves its tolerability. In addition, the organization of nutrition of patients receiving PTP, it is necessary to use sugar, which will improve the tolerability of chemotherapy using isoniazid [5]. Daily coadministration of isoniazid, pyrazinamide and rifampicin led to the development of severe hepatotoxic reactions by 30 days of the experiment, manifested in the change of a number of clinically significant indicators, in particular, an increase in ALT, AST in blood serum by 3 times (table), as well as a decrease in their activity in liver tissue homogenates compared with the control group [6] (Table 1).

Table 1: Dynamics of the content of ALT and AST in serum of rats when the drug to lose the liver of rats PTP with AM and without $(\mathrm{n}=120)$.

\begin{tabular}{|c|c|c|c|c|}
\hline \multirow{2}{*}{$\begin{array}{l}\text { The Determined } \\
\text { Parameters }\end{array}$} & \multirow{2}{*}{$\begin{array}{l}\text { Preparations and Duration of their } \\
\text { Administration }\end{array}$} & \multicolumn{3}{|c|}{ Terms of Registration of Indicators } \\
\hline & & 3 day & 30 day & 60 day \\
\hline \multirow[t]{4}{*}{ ALT, U/l } & $\begin{array}{l}\text { PTP from 1-30 days, AM from 31-60 days after } \\
\text { discontinuation of PTP }\end{array}$ & $87,6 \pm 0,5^{* * *}$ & $191,2 \pm 6,6^{* * *}$ & $136,0 \pm 5,2^{* * *}$ \\
\hline & PTP AM, with 1-60 day & $67,2 \pm 2,1^{*}$ & $71,2 \pm 4,1^{*}$ & $79,6 \pm 3,9^{* *}$ \\
\hline & UM, $1-60$ days. & $60,9 \pm 0,7$ & $59,2 \pm 1,8$ & $62,2 \pm 3,4$ \\
\hline & Dynamic control & $61,2 \pm 1,5$ & $61,8 \pm 2,0$ & $66,2 \pm 1,9$ \\
\hline \multirow[t]{4}{*}{ AST, U/l } & $\begin{array}{l}\text { PTP from 1-30 days, AM from 31-60 days after } \\
\text { discontinuation of PTP }\end{array}$ & $68,2 \pm 2,2^{*}$ & $172,4 \pm 13,2^{* * *}$ & $129,2 \pm 7,3^{* * *}$ \\
\hline & PTP AM, with 1-60 day & $56,7 \pm 6,0$ & $64,9 \pm 4,1$ & $71,2 \pm 3,7^{*}$ \\
\hline & UM, 1-60 days. & $55,2 \pm 2,9$ & $52,8 \pm 2,7$ & $57,1 \pm 5,1$ \\
\hline & Dynamic control & $57,2 \pm 4,2$ & $55,0 \pm 3,3$ & $58,4 \pm 3,2$ \\
\hline
\end{tabular}

Note: the level of significance of differences in relation to the values of dynamic control, at $\quad{ }^{*} \mathrm{p}<0.05 ;{ }^{* *} \mathrm{p}<0.01 ;{ }^{* * *} \mathrm{p}<0.001$.

An increase in serum enantiospecific enzyme urokinase 9.6 times, phospholipase and 3.6 times, alkaline phosphatase 1.3 times compared to dynamic control. When administered from 1-30 days PTP rats GGT level (gamma-glutamyl transpeptidase) in serum decreased by $34 \%$. Cancellation of the introduction of PTP, starting from 31 days of the experiment, and the subsequent appointment of AM, within 30 days, did not lead to a complete restoration of the estimated indicators. Joint administration of AM with PTP, from 1 to 60 days of the experiment, prevented the development of severe drug complications on the 30th day of the experiment, which allowed to continue continuous administration of PTP up to 60 days [6]. The results of the study suggest that the use of AM, starting from the first days of taking PTP, can reduce the hepatotoxicity of PTP used. 


\section{Summary}

i) The use of PTP, taking into account the influence of circadian rhythms on their tolerability, allows to reduce the undesirable effects produced by them.

ii) The appointment of PTP after a meal with the addition of sugar to the diet improves the tolerability of drugs.

iii) The combined use of PTP and AM can reduce the manifestations of NPE PTP, which can increase the effectiveness of chemotherapy for pulmonary tuberculosis.

iv) The obtained data are recommended for use in clinical practice.

\section{References}

1. Guskova TA (2008) Toxicology of drugs. MPI pp. 196.

\section{ISSN: 2574-1241}

DOI: 10.26717/BJSTR.2019.19.003320

Usov KI. Biomed J Sci \& Tech Res

This work is licensed under Creative Commons Attribution 4.0 License

Submission Link: https://biomedres.us/submit-manuscript.php
2. Usov KI, Guskova TA, Yushkov GG (2018) The Role of pyridoxine hydrochloride in the development of animal tolerance to the toxic effects of isoniazide. Tuberculosis and lung disease. 96(6): 51-57.

3. Sukhanov DS, Vinogradova TI, Zabolotnykh N In, Vitovskaya ML, Kovalenko AL (2014) Hepatotropic action of ranikhola and ademetionine damage liver anti-TB drugs the main series in the experiment. Archives of pathology 76(2): 26-31.

4. Usov KI, Guskova TA, Yushkov GG, Mashanov AV, Gushchina AA (2017) Influence of chronobiological rhythms on toxicity of isoniazid and rifampicin in experimental conditions on rats. Toxicological Bulletin 4: 24-33.

5. Usov KI, Guskova TA, Yushkov GG, Guschina AA (2018) The Effect of sucrose and pyridoxine hydrochloride on the toxicity of isoniazide in an acute experiment. Tuberculosis and socially significant diseases $3: 14-$ 18.

6. Usov KI, Guskova TA, Yushkov GG, Grishina LP, Gushchin AS (2018) Experimental substantiation of maintenance therapy with the use of ademetionine to reduce hepatotoxic reactions of anti-tuberculosis drugs. Toxicological Bulletin 6: 12-21.

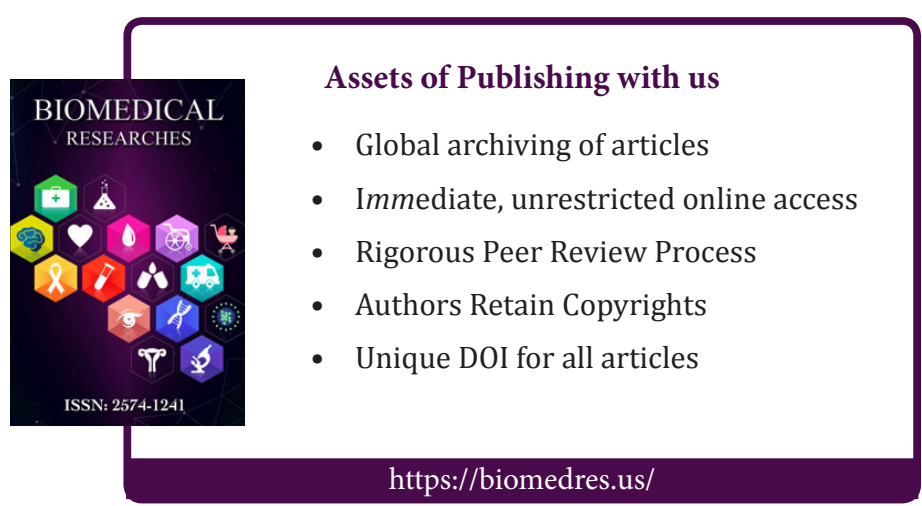

